\title{
The Tool Switching Problem Revisited
}

\author{
Yves Crama \\ HEC Management School, University of Liège, Boulevard du Rectorat, 7 (B31), B-4000 \\ Liège, Belgium, Y.Crama@ulg.ac.be \\ Linda S. Moonen, Frits C.R. Spieksma \\ Department of Applied Economics, Katholieke Universiteit Leuven, Naamsestraat 69, \\ B-3000 Leuven, Belgium, \{linda.moonen|frits.spieksma\}@econ.kuleuven.be \\ Ellen Talloen \\ PricewaterhouseCoopers, Woluwe Garden, Woluwedal 18, B-1932 Sint-Stevens-Woluwe, \\ Belgium, ellen.talloen@pwc.be
}

In this note we study the complexity of the tool switching problem with non-uniform tool sizes. More specifically, we consider the problem where the job sequence is given as part of the input. We show that the resulting tooling problem is strongly NP-complete, even in case of unit loading and unloading costs. However, we show that if the capacity of the tool magazine is also given as part of the input, the problem is solvable in polynomial time.

\section{Introduction}

Consider the following problem: given is a set of jobs $J$, a set of tools $T$, and a single machine equipped with a tool magazine of size $C$. The machine is only capable of performing job $j \in J$ when a given subset of the tools $T(j) \subseteq T$ is present in the magazine of the machine. Each tool $t \in T$ has a size which represents the number of slots occupied by the tool in the magazine. Since the capacity required to store all tools in $T$ in the magazine exceeds $C$, tools need to be inserted in and removed from the magazine in order to process all jobs. It is assumed that inserting and removing a tool is only allowed in between the processing of a pair of consecutive jobs, and that all tools in $T(j)$ need to be present in the magazine before job $j \in J$ can start. We refer to inserting a tool in the magazine as a loading operation, and to removing a tool from the magazine as an unloading operation. The tool switching problem is now to find a sequence of the jobs, and an associated sequence of tool loadings, that minimizes the total number of loading and unloading operations (L/U operations).

In this note we focus on the problem that results when the sequence of the jobs is given; we refer to this problem as the tooling problem (TP). The basic tooling problem assumes unit costs for L/U operations, and uniform tool sizes (meaning that each tool occupies a same number of slots of the magazine). Here, we consider the case of non-uniform tool sizes; nonuniform tool sizes relate to the situation where this number need not be the 
same for each tool. This is often the case in practice (see for instance Stecke (1983), Jain et al. (1996) or Matzliach and Tzur (2000)). A difficulty that arises when considering non-uniform tool sizes is that the physical location of a tool in the magazine becomes relevant. Observe that when all tools have size 1, the only relevant decision is whether the tool is in the magazine or not; if tool sizes are non-uniform, the location of a tool in the magazine becomes important. For instance, removing two tools of size one from nonadjacent slots does not create enough space to load a tool of size two. Thus, in addition to determining which tools need to be present in the magazine, one now must also specify its location in the magazine.

\subsection{Related Work}

The general tool switching problem was first considered by Tang and Denardo (1988). (It is interesting to note that a special case of the tool switching problem was already considered by Belady (1966) in the context of the socalled paging problem; the special case arises since in this application each job needs a single tool). The tool switching problem is already NP-hard for $C=2$ (see Crama et al. (1994)). Many heuristics have been proposed for its solution. Recent papers are from Djellab et al. (2000), Song and Hwang (2002), Tzur and Altman (2004), and Zhou et al. (2005). The approximability of the problem is shortly discussed in Crama and van de Klundert (1999). Solving large instances of the tool switching problem to optimality is a challenge: Laporte et al. (2004) report on solving instances with up to 25 jobs and 25 tools, which are the largest solved instances we are aware of.

For a fixed sequence of the jobs, Tang and Denardo (1988) proposed a procedure called KTNS (Keep Tools Needed Soonest), and gave an ad-hoc proof of its correctness (thereby generalizing Belady's result) for the basic TP. Crama et al. (1994) provided an alternative proof of the correctness using interval matrices, which allowed a generalization when an arbitrary setup $\operatorname{cost} b_{t}$ is given for each tool $t \in T$. These results have been further generalized to the case of changeover costs of the form $d_{i k}$ when tool $i$ is inserted directly after removing tool $k$ by Privault and Finke (1995). They showed that in case of these changeover costs problem TP can still be solved in polynomial time by formulating it as a min-cost flow problem. All these results apply to uniform tool sizes.

So far, relatively little attention is paid to the tool switching problem with non-uniform tool sizes. Matzliach and Tzur (2000) show that this problem is NP-complete by a reduction from Partition, thereby resolving an open question posed by Crama (1997). A drawback of their reduction is that the physical location of the tools in the magazine is ignored; moreover, their 
result does not imply strong NP-completeness. Tzur and Altman (2004) propose a heuristic for solving the tool switching problem with a fixed job sequence with non-uniform tool sizes, KSTNS (Keep Smallest Tools Needed Soonest), which is a modification of the KTNS-procedure proposed by Tang and Denardo (1988). Unlike the KTNS-procedure, KSTNS is not guaranteed to produce an optimal solution.

In literature, two types of magazines are described. One type is the socalled straight magazine which essentially is a row of consecutive slots. This type of magazine is very common in PCB assembly. The other type is a round magazine, which is commonly used in the metal-based industry (see Tzur and Altman (2004)). Our results hold for both cases.

\subsection{Results}

We present two results in this note:

- TP is strongly NP-complete, even with unit loading and unloading costs. We use a reduction from 3-Partition in which the physical location of the tools in the magazine plays a crucial role (see Section 2).

- For a fixed value of $C$, TP is solvable in polynomial time. It turns out that when $C$ is part of the input, a shortest path computation on a network involving $O\left(|T|^{C} C\right.$ !) nodes gives the minimum number of $\mathrm{L} / \mathrm{U}$ operations (see Section 3).

\section{Complexity}

In this section we show that $\mathrm{TP}$ with a straight magazine is NP-complete in the strong sense, even in case of unit loading and unloading costs. We use a reduction from 3-Partition, which is known to be strongly NP-complete (Garey and Johnson (1979)). The problem 3-Partition can be defined as follows:

\section{3-PARTition}

Given a set $S$ of $3 n$ elements, a positive integer $B$ (we assume that $B$ is even), and integral weights $w_{k} \geq 0$ for all elements $k \in S$ such that $B / 4<w_{k}<B / 2$ and such that $\sum_{k \in S} w_{k}=n B$, the problem is to partition $S$ into $n$ disjoint triples $T_{1}, \ldots, T_{n}$ such that, for $1 \leq i \leq n, \sum_{k \in T_{i}} w_{k}=B$.

Now, given an instance $\mathcal{I}$ of 3 -Partition, we define an instance $\mathcal{I}^{\prime}$ of TP with the following characteristics: 
- The capacity of the tool magazine $C=2 n(B / 2+1)$.

- The number of jobs equals $2 n+2$.

- The number of tools equals $9 n$, and their sizes are given in Table 1.

- All loading operations and all unloading operations have cost equal to 1 .

- The sets $T(j), j=1, \ldots, 2 n+2$, are specified as follows:

- Job 1 needs tools $t_{1}, \ldots, t_{2 n}$ (each with size $B / 2+1$ ).

- Job $j$ needs tools $t_{1}, \ldots, t_{2 n-j+1}$, (with size $B / 2+1$ ), tools $t_{2 n+1}, \ldots, t_{2 n+j-1}$ (with size $B / 2$ ), and tools $t_{4 n+1}, \ldots, t_{4 n+j-1}$ (with size 1 ), for $j=2, \ldots, 2 n+1$.

- Job $2 n+2$ needs tools $t_{4 n+1}, \ldots, t_{6 n}$ (with size 1 ), and tools $t_{6 n+1}, \ldots, t_{9 n}$ (with sizes corresponding to the weights from problem instance $\mathcal{I}$ from 3-Partition).

Table 1: Tool sizes for problem instance $\mathcal{I}^{\prime}$

\begin{tabular}{c|c}
\hline Tools & Size \\
\hline$t_{1}, \ldots, t_{2 n}$ & $B / 2+1$ \\
\hline$t_{2 n+1}, \ldots, t_{4 n}$ & $B / 2$ \\
\hline$t_{4 n+1}, \ldots, t_{6 n}$ & 1 \\
\hline$t_{6 n+1}, \ldots, t_{9 n}$ & $w_{k}, k \in S$ \\
\hline
\end{tabular}

We can now formulate the decision problem corresponding to TP:

Decision problem: Does there exist a solution to instance $\mathcal{I}^{\prime}$ of problem TP with no more than $13 n \mathrm{~L} / \mathrm{U}$ operations?

In order to show NP-completeness, we show that a YES-instance of 3Partition corresponds to a YES-instance of TP, and vice versa.

- First we assume that we have a YES-instance for 3-Partition, meaning that there exists a solution consisting of $n$ triples, each with weight equal to $B$. Consider the following solution to TP: to perform job 1 , we load tools $t_{1}, \ldots, t_{2 n}$ consecutively in the tool magazine. For job 2 we remove $t_{1}$ and replace it by placing $t_{4 n+1}$ in slot 1 and $t_{2 n+1}$ in slots $2, \ldots, \frac{1}{2} B+1$. For job 3 , we replace $t_{2}$ by tools $t_{2 n+2}$ in slots $B / 2+2, \ldots, B+1$, and $t_{4 n+2}$ in slot $B+2$. Notice that the two tools $t_{2 n+1}$ and $t_{2 n+2}$ with size $B / 2$ are placed next to each other. In a similar way we deal with jobs $4, \ldots, 2 n+1$. For the final job (job $2 n+2$ ) 
we unload all tools with size $B / 2$ from the magazine, leaving $n$ empty series of slots of size $B$. We can load the required tools $\left(t_{6 n+1}, \ldots, t_{9 n}\right)$ into the magazine in such a way that each empty series of slots of size $B$ contains 3 tools. This is possible since we know that there exists a solution to the instance of 3-Partition. The number of $\mathrm{L} / \mathrm{U}$ operations corresponding to this solution is equal to $2 n$ (for job 1 ) $+3 \times 2 n$ (for jobs $2, \ldots, 2 n+1)+2 n+3 n$ (for job $2 n+2)=13 n$, so we conclude that we have a YES-instance for TP.

- Now let's assume that we have a YES-instance for TP, so we have a solution to TP with no more than $13 n \mathrm{~L} / \mathrm{U}$ operations. In order to process job 1 , we must load tools $t_{1}, \ldots, t_{2 n}$ in the magazine; then, for processing job $j$, for $j=2, \ldots, 2 n+1$, we must unload tool $t_{2 n-j+2}$ (i.e., a tool of size $B / 2+1$ ) and we must load tools $t_{2 n+j-1}($ size $B / 2$ ) and $t_{4 n+j-1}$ (size 1) in the slots which have just be emptied. This requires at least $2 n+3 \times 2 n=8 n \mathrm{~L} / \mathrm{U}$ operations, no matter how the tools are placed in the magazine. Then, for job $2 n+2$, we must unload all tools $t_{2 n+1}, \ldots, t_{4 n}$ of size $B / 2$ and we must load all tools of size $w_{1}, \ldots, w_{3 n}$. This amounts to $5 n \mathrm{~L} / \mathrm{U}$ operations for the last job.

Hence, if a solution with cost $13 n$ exists, this solution cannot involve any additional L/U operations beyond those which have just been listed, meaning that we are not allowed to displace any other tool in the process.

In particular, this analysis implies that when job $2 n+1$ is processed, the magazine contains $2 n$ tools of size $B / 2$ and $2 n$ tools of size 1 , and each tool of size $B / 2$ is adjacent to at least one tool of size 1 . Let us call a tool of size $B / 2$ isolated if it is adjacent to two tools of size 1 (as shown in configuration (b) in Figure 1), or if this tool is the leftmost or rightmost tool in the magazine. We claim that that there is no such isolated tool in a solution with $13 n \mathrm{~L} / \mathrm{U}$ operations.

a)

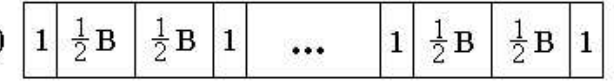

b)

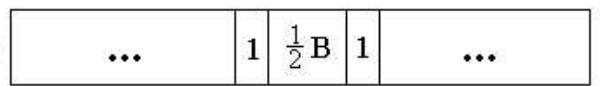

Figure 1: Possible ways in which the tool magazine can be filled.

After finishing job $2 n+1$, there are two possibilities: either there are no isolated tools, as shown in configuration (a) in Figure 1, or there is at least one isolated tool of size $B / 2$, as shown in configuration (b) in Figure 1. 
We first analyze the second case (corresponding to configuration (b) in Figure 1): the isolated tool of size $B / 2$ needs to be removed from the magazine before we can start the final job. In this empty space, we can add some of the tools that are required for this job. We know that at least one of these required tools will fit, since $w_{k}<B / 2$ for all $k \in S$. However, we also know that $w_{k}>B / 4$ for all $k \in S$, meaning that we will never be able to fit 2 tools in this empty space. This means that, in order to fill the magazine to contain all tools required for job $2 n+2$, we need additional $\mathrm{L} / \mathrm{U}$ operations, resulting in more than $13 n \mathrm{~L} / \mathrm{U}$ operations in total. Hence configuration (b) cannot occur.

In the case depicted in configuration (a) in Figure 1 however, we have the following: after finishing job $2 n+1$, we remove all tools of size $B / 2$, leaving $n$ empty spaces of size $B$. Since we have a solution to TP using $13 n \mathrm{~L} / \mathrm{U}$ operations, we know that we can add the required $3 n$ tools using only $3 n \mathrm{~L} / \mathrm{U}$ operations. Now, since $B / 4<w_{k}<B / 2$ for all $k \in S$, we know that each empty space of size $B$ can fit more than 2 tools, but never 4 . This means that, in order to fill the magazine using at most $3 n \mathrm{~L} / \mathrm{U}$ operations, each of these spaces must contain exactly 3 tools. This means that the problem instance corresponding to 3-Partition must be a YES-instance.

The above analysis shows that the tooling problem is strongly NP-complete. We leave it to the reader to verify that a similar reasoning applies to a round layout of the tool magazine. However, in the next section we show that if the capacity of the tool magazine is fixed (i.e., if $C$ is given in the input of the problem), the problem is solvable in polynomial time.

\section{Fixed value of $\mathrm{C}$}

In this section we consider the tooling problem for a fixed value of the tool magazine capacity. In other words, we assume that the capacity $C$ is given in the input of the problem. As we will show in this section, the resulting problem is solvable in polynomial time.

First we introduce some notation. Given are the set $T$ containing all the tools, plus $C$ dummy tools of size 1, which represent empty slots in the tool magazine. We call $T^{\prime}$ the set of all tools in $T$ together with all dummy tools. Then, a magazine configuration can be described by listing at most $C$ elements of $T^{\prime}$. For example, the configuration $\left\{t_{4}, t_{1}, t_{d}, t_{2}, t_{d}\right\}$ corresponds to the magazine shown in Figure 2, where $t_{d}$ represent dummy nodes (i.e., empty spaces in the tool magazine). Observe that, if we have $\left|T^{\prime}\right|$ tools, there are at most $O\left(\left|T^{\prime}\right|{ }^{C} C\right.$ !) different magazine configurations. 


\begin{tabular}{|l|l|l|l|l|}
\hline $\mathbf{t}_{4}$ & $\mathrm{t}_{1}$ & $\mathrm{t}_{\mathrm{d}}$ & $\mathbf{t}_{2}$ & $\mathbf{t}_{\mathrm{d}}$ \\
\hline
\end{tabular}

Figure 2: Possible configuration for the tool magazine.

Now we create a directed graph $D=(V, A)$ containing $n$ layers of vertices ( $n$ being the number of jobs). A vertex in layer $\ell$ of $D$ corresponds to a feasible magazine configuration for performing job $\ell$, so there are at most $O\left(\left|T^{\prime}\right|{ }^{C} C\right.$ !) vertices in each layer (notice that there is a vertex only for those magazine configurations that contain all required tools for a specific job). The arc set $A$ contains arcs from all vertices in layer $\ell$ to all vertices in layer $\ell+1(\ell=1, \ldots, n-1)$, and an arc $(i, j)$ has a length equal to the switching costs to go from the magazine configuration corresponding to vertex $i$ to the magazine configuration corresponding to vertex $j$. In order to find an optimal solution to the tooling problem, we need to find a shortest path from layer 1 to layer $n$ in the resulting graph $D$. This can be done in polynomial time in case of a fixed value of the magazine capacity $C$.

Notice that the only thing required is to be able to specify the switching costs between two magazine configurations. Also, the method described in this section holds for a straight tool magazine, as well as for a round magazine.

\section{Conclusion}

In this paper, we consider a special case of the tool switching problem, where the tools have non-uniform sizes, and the job sequence is given. We show that the resulting tooling problem is strongly NP-complete, even for unit loading and unloading costs. We also show that, in case the capacity of the tool magazine is also given in the problem input, the problem can be solved in polynomial time by solving a shortest path problem in a directed graph. 


\section{References}

Belady, L.A. (1966). A study of replacement algorithms for a virtual-storage computer. IBM Systems Journal 5, 78-101.

Crama, Y. (1997). Combinatorial optimization models for production scheduling in automated manufacturing systems. European Journal of Operational Research 99, 136-153.

Crama, Y., J. van de Klundert (1999). Worst-case performance of approximation algorithms for tool management problems. Naval Research Logistics 46, 445462.

Crama, Y., A.W.J. Kolen, A.G. Oerlemans, F.C.R. Spieksma (1994). Minimizing the number of tool switches on a flexible machine. The International Journal of Flexible Manufacturing Systems 6, 33-54.

Djellab, H., K. Djellab, M. Gourgand (2000). A new heuristic based on a hypergraph representation for the tool switching problem. International Journal of Production Economics 64, 165-176.

Garey, M.R., D.S. Johnson (1979). Computers and intractability. A guide to the theory of NP-completeness. W.H. Freeman and Company, New York.

Jain, S., M.E. Johnson, F. Safai (1996). Implementing setup optimization on the shop floor. Operations Research 43, 843-851.

Laporte, G., J.-J. Salazar, F. Semet (2004). Exact algorithms for the job sequencing and the tool switching problem. IIE Transactions 36, 37-45.

Matzliach, B., M. Tzur (2000). Storage management of items in two levels of availability. European Journal of Operational Research 121, 363-379.

Privault, C., G. Finke (1995). Modelling a tool switching problem on a single NC-machine. Journal of Intelligent Manufacturing 6, 87-94.

Stecke, K.E. (1983). Formulation and solution of nonlinear integer production planning problems for flexible manufacturing systems. Management Science $29,273-288$.

Song, C.Y., H. Hwang (2002). Optimal tooling policy for a tool switching problem of a flexible machine with automatic tool transporter. International Journal of Production Research 40, 873-883.

Tang, C.S., E.V. Denardo (1988). Models arising from a flexible manufacturing machine, part I: minimization of the number of tool switches. Operations Research $36,767-777$.

Tzur, M., A. Altman (2004). Minimization of tool switches for a flexible manufacturing machine with slot assignment of different tool sizes. IIE Transactions 36, 95-110.

Zhou, B.H., L.F. Xi, Y.S. Cao (2005). A beam-search-based algorithm for the tool switching problem on a flexible machine. International Journal of Advanced Manufacturing Technology 25, 876-882. 